\section{SURGICAL APPROACH TO AORTIC VALVE DISEASE WITH CONCOMITANT DILATION OF THE PROXIMAL AORTA}

To the Editor:

We read with interest the excellent article by Lee and colleagues, ${ }^{1}$ in which an aggressive surgical strategy toward concomitant dilatation of the proximal aorta in patients with aortic valve disease was not recommended, even in the presence of moderately dilated aorta. We agree with their conclusions up to a point, and we therefore would like to report our personal experience in a much smaller cohort of 38 patients with slightly to moderately dilated proximal aortas (from 40 to $55 \mathrm{~mm}$ ) and aortic valve disease who for several reasons were submitted to just isolated aortic valve replacement and were subsequently followed-up (median duration 36 months). ${ }^{2}$

Briefly, we identified 3 different behaviors of the proximal aorta during the follow-up: (1) no significant change, (2) increased growth velocity of the aortic diameter without reaching the limit of $55 \mathrm{~mm}$ (the commonly accepted indication to an ascending aorta replacement), and (3) increase of the proximal aorta reaching 55 $\mathrm{mm}$ or actual undergoing rupture or dissection. Of 36 early survivors, 3 fell into this third group $(8.3 \%$; linearized incidence of $2.3 \% /$ patient-y), because either the aortic diameter reached $55 \mathrm{~mm}$ or larger, precipitating elective reoperation (2 cases) or acute aortic dissection occurred (1 case).

In our regression model, a steep increase in the aortic expansion rate was correlated with a preoperative diameter of $50 \mathrm{~mm}$ or more $(R=0.83$; $P=.0001)$. We therefore advocated replacement (or at least a reduction plasty) of the ascending aorta at a level of approximately 48 to $50 \mathrm{~mm}$, even in patients with no additional risk factors, such as the presence of a bicuspid aortic valve or Marfan syndrome.
Because the combined operation can be performed without significant additional risks while carrying the important advantage of eliminating the threat of later reoperations or acute aortic syndromes, we believe that an aggressive approach is reasonable when dealing with the common setting of concomitant aortic valve and proximal aorta disease. Patients with additional risk factors should probably be submitted to ascending aorta replacement even at lower levels of dilation (approximately 40-45 $\mathrm{mm}$ ).

Probably a less aggressive strategy might be justified in cases of isolated aortic stenosis with mild dilatation of the ascending aorta. From our standpoint, in cases of aortic stenosis, the immediate resolution of the fluid dynamics abnormality that led to aortic expansion, obtained with the valve replacement, might be sufficient to prevent further dilatation; on the other hand, in patients with prevalent aortic regurgitation, the volume overload still persists after valve replacement, perpetuating the hemodynamic harm to the aortic wall. Actually, in our series the aortic growth velocity was $-0.6 \pm 1.5 \mathrm{~mm} / \mathrm{y}$ in patients with prevalent stenosis and $+1.4 \pm$ $4.8 \mathrm{~mm} / \mathrm{y}$ in patients with prevalent regurgitation. This difference was, however, only marginally significant $(P=.10)$. Indeed, in the group with isolated aortic valve replacement, the expansion rate reported by Lee and colleagues $^{1}$ is exactly the same described by us in the aortic stenosis group of our series. We therefore wonder whether they tried to identify a different behavior in the expansion rate of patients with stenosis versus those with prevalent regurgitation. Perhaps different approaches to these distinct conditions would be reasonable.

\footnotetext{
Carlo Bassano, $M D, P h D$ Emanuele Bovio, $M D$ Luigi Chiariello, $M D$ Cardiac Surgery
}

\section{Tor Vergata University}

Rome, Italy

\section{References}

1. Lee SH, Kim JB, Kim DH, Jung SH, Choo SJ, Chung $\mathrm{CH}$, et al. Management of dilated ascending aorta during aortic valve replacement: valve replacement alone versus aorta wrapping versus aorta replacement. J Thorac Cardiovasc Surg. 2013;146:802-9.

2. Bassano C, Fratticci L, Del Giudice C, Andò G, De Paulis R, Nardi P, et al. Ectasia of the ascending aorta at the time of aortic valve surgery: replace or relax? It Heart J. 2005;6:968-71.

http://dx.doi.org/10.1016/ j.jtcvs.2013.10.080

\section{VARIOUS SURGICAL APPROACHES TO CORONARY ARTERY ANEURYSM \\ To the Editor:}

We read with great interest the article by Daralammouri and colleagues $^{1}$ on surgical treatment of a giant right coronary artery aneurysm. In this report, they described surgical treatment including resection of aneurysm and proximal-distal ligation with coronary artery bypass grafting in the proximal huge right coronary aneurysm. We congratulate Daralammouri and colleagues ${ }^{1}$ on their successful treatment. We would also like to add a short comment about the choice of surgical therapy.

Surgical intervention is a reasonable approach because of the morphologic variability and enlarged size of these aneurysms. The main purpose of surgical treatment in coronary artery aneurysms is to prevent their rupture and the patient's death, as well as to avoid thrombosis and related coronary embolization. ${ }^{2,3}$

There are various surgical approaches, including resection and plication of the aneurysm, graft interposition, end-to-end anastomosis technique, and ligation with distal bypass grafting. ${ }^{2,3}$ The surgical technique is still controversial, and there is no consensus regarding the optimal surgical strategy. 